\section{Automonitoreo de la glucemia en pacientes con diabetes tipo 2: la opinión de los pacientes}

Los especialistas consideran el autocontrol como la piedra angular para el éxito del control de la diabetes y el automonitoreo de los niveles de glucemia desempeña un importante papel en ese sentido. En el caso de la diabetes tipo 2, sin embargo, no hay un consenso total al respecto entre los médicos y en la literatura científica. En ese contexto, es importante conocer la opinión de los pacientes - ya que son los responsables de realizar las mediciones y tomar las acciones necesarias diariamente- $-\mathrm{y}$ ejecutar en cuenta los elementos que ellos consideran positivos o negativos. Por ejemplo, se ha visto que a pesar de que el automonitoreo podría mejorar la conciencia sobre el efecto de la dieta en los niveles de glucemia, también produce un sentimiento de fracaso si las mediciones se mantienen altas, lo que pudiera llevar al abandono de las dietas recomendadas.

Para conocer la opinión de los pacientes con diagnóstico de diabetes tipo 2 acerca del automonitoreo de los niveles de glucemia en el tiempo y los signos de "fatiga" respecto al automonitoreo, se realizó un estudio cualitativo longitudinal en centros de atención primaria y secundaria de Lothian, Escocia, Reino Unido.

Se observó que la constancia en el automonitoreo disminuyó con el tiempo. Los tres factores principales que emergieron de este estudio fueron: el papel de desestimulación desempeñado por los médicos, las dificultades para interpretar los resultados y definir la respuesta que exigen las lecturas, y la relación personal de los pacientes con el automonitoreo.

En el primer caso, los participantes se refirieron al poco interés que mostraban los médicos y otros profesionales sanitarios en los niveles medidos de glucemia, ya que concentraban más su atención en los niveles de hemoglobina $\mathrm{A}_{1 \mathrm{c}}$. Esta conducta se interpretaba como un indicador de la poca utilidad del automonitoreo.

Acerca del segundo factor, algunos pacientes seguían encontrando dificultades para realizar las lecturas de la glucemia e interpretar sus resultados, así como para decidir qué medidas de respuesta debían tomar. No se encontró evidencia de que los participantes se guiaran por los resultados del automonitoreo para decidir cambios de conducta.
Esto podría explicar por qué no se ha encontrado una asociación estable entre el automonitoreo y el control de la glucemia.

Por último, las lecturas consistentemente estables y el mejor conocimiento de los indicadores físicos de la hiperglucemia también causaron un relajamiento de la atención al automonitoreo. Algunos pacientes veían las lecturas como un reflejo de su buena o mala conducta y se reprimían a sí mismos, especialmente las mujeres. Los pacientes que atribuían sus resultados a causas externas (como la falta de medicamentos) eran más propensos a mantener el automonitoreo. Conservar la tranquilidad y el hábito adquirido fueron dos razones clave para mantener el automonitoreo.

A pesar de que este estudio se limitó a analizar las opiniones de los pacientes diabéticos acerca del automonitoreo de la glucemia, los autores afirman que los resultados pueden aplicarse a otras situaciones en las que los pacientes administren algún tipo de tecnología para controlar su salud. Las fortalezas de este estudio se basan en que se tomaron en cuenta los puntos de vista tanto de pacientes recién diagnosticados como de otros ya veteranos; además, el estudio se basó en una perspectiva longitudinal exhaustiva y se enfocó en el automonitoreo en general, sin desviar la atención de los pacientes hacia su enfermedad o hacia los servicios de salud. El estudio también tiene debilidades, fundamentalmente por depender de la opinión de los pacientes y no tomar en cuenta las mediciones objetivas de la glucemia ni la opinión de los profesionales sanitarios. Aunque se trató de seleccionar una muestra representativa de los diversos segmentos de la población de pacientes diabéticos de la ciudad, como ocurre en toda investigación cualitativa no es posible generalizar los resultados indiscriminadamente.

No obstante, es un hecho que la incertidumbre clínica acerca de la posible eficacia del automonitoreo y el papel que este puede desempeñar en el control de la glucemia en la diabetes tipo 2 se refleja en la opinión de los pacientes participantes. Los pacientes no actuaron en respuesta a los resultados del automonitoreo, en parte por la falta de educación al respecto. Los profesionales de la salud deben explicar claramente cuándo los pacientes deben monitorear su nivel de glucemia, cómo interpretar las lecturas y qué acciones deben emprender, especialmente cuando las lecturas son altas. La acti- 
tud de los médicos y de otros profesionales de la salud es un factor crítico en ello y deben tener en cuenta que los pacientes con la diabetes controlada ven el automonitoreo más positivamente que los pacientes con signos de descompensación.

Las investigaciones futuras deben explorar la actitud de los profesionales de la salud con respecto al automonitoreo y examinar la información y las orientaciones que al respecto se dan a los pacientes en las consultas. Se debe hacer un especial énfasis en establecer las características que pueden ayudar a predecir cuáles pacientes pueden confundirse con este procedimiento y cuáles pueden sacar beneficios para mejorar su salud y su calidad de vida. La educación sobre el automonitoreo de la glucemia debe ser explícita y continua, y estar orientada a alcanzar sus objetivos concretos, particularizada según las características y necesidades de cada paciente. (Peel E, Douglas M, Lawton J. Self monitoring of blood glucose in type 2 diabetes: longitudinal qualitative study of patients' perspectives. Br Med J. 2007;335:493.)

\section{¿Puede la transmisión de la gripe aviar A subtipo H5N1 de persona a persona provocar una epidemia?}

El virus de la gripe aviar A de alta patogenicidad (IAAP) subtipo H5N1 ha traspasado en reiteradas ocasiones la barrera de la especie y ha afectado a personas. Desde diciembre de 2003 se han informado 291 casos de IAAP (H5N1) en personas, con un desenlace de 172 muertes (tasa de letalidad de $59,1 \%$ ) en 12 países, principalmente del sudeste asiático. Entre esos casos se han documentado 31 conglomerados familiares, compuestos por 2-8 personas relacionadas por vínculos en el hogar. Hasta el momento no se ha establecido cuántos de esos casos se deben a una fuente aviar común o si ha habido también transmisión de persona a persona. $\mathrm{Si}$ alguna o algunas cepas de IAAP del subtipo H5N1 adquieren la capacidad de transmitirse sostenidamente de persona a persona, el brote resultante podría extenderse a todo el mundo - por la red de transporte internacional- a mayor velocidad de lo que tomaría desarrollar y distribuir una vacuna específica y efectiva contra la nueva variante viral.

En este trabajo se analizan los datos de dos de los mayores conglomerados familiares de gripe aviar del subtipo H5N1 con el objetivo de determinar si ha habido transmisión de persona a persona $\mathrm{y}$, en caso positivo, el grado de transmisibilidad de esa cepa. Se estudiaron ocho casos que formaron un conglomerado familiar en el norte de Sumatra, Indonesia, entre abril y mayo de 2006 y otro conglomerado familiar de ocho casos confirmados de
H5N1, detectados en el distrito de Dogubayazit, en la región oriental de Turquía, entre el 18 de diciembre de 2005 y el 15 de enero de 2006.

Se encontró que el período de incubación en el brote de Indonesia fue posiblemente de 3-7 días y el período infeccioso más probable fue de 5-13 días. Se encontró evidencia de transmisión de persona a persona $(P=0,009)$. La tasa estimada de síndrome respiratorio agudo (SRA) en el hogar fue de 0,29 (intervalo de confianza de 95\% [IC95\%]: 0,15 a 0,51 ).

En el brote ocurrido en Turquía no se demostró que la infección se hubiera transmitido de persona a persona $(P=0,114)$ y la probabilidad diaria de infección a partir de una misma fuente fue de 0,011 (IC95\%: 0,005 a 0,025).

De esta manera, se encontró respaldo estadístico para afirmar que hubo diseminación de la cepa de IAPP del subtipo H5N1 de persona a persona en el hogar estudiado en Sumatra, Indonesia, y que la tasa de SRA intrafamiliar (la probabilidad de que una persona infectada haya transmitido la infección a otro miembro del hogar) fue de $29 \%$. Esta tasa es similar a los estimados estadísticos obtenidos para la gripe A interpandémica en los Estados Unidos de América (12,7\%-30,6\%). El tiempo promedio de incubación de esta cepa (aproximadamente 5 días) es el doble del observado en las cepas aisladas durante la última pandemia de gripe y la actual etapa interpandémica.

Sin embargo, aunque se haya demostrado que ocurrió la transmisión de persona a persona, no está claro que el virus pueda mantener una transmisión interpersonal sostenida. Además, es posible que para la transmisión de este virus se requiera un contacto muy estrecho con la persona infectada. Así, incluso sin intervención alguna, la probabilidad de que la transmisión de la infección a una única persona desencadene la diseminación del virus es solo de aproximadamente $12 \%$ con las cepas estudiadas. Sin embargo, es importante tomar en cuenta que el uso profiláctico de oseltamivir haya contribuido a limitar la diseminación de la infección y que, aunque no se obtuvo un respaldo estadístico de la transmisión de persona a persona en el caso estudiado de SRA intrafamiliar en Turquía, no se puede excluir la posibilidad de que haya ocurrido.

Mediante sistemas automatizados de simulación se ha podido demostrar que con el empleo dirigido de agentes antivirales se podría contener la diseminación de una cepa pandémica de gripe si se logra comenzar su aplicación en el transcurso de las tres semanas siguientes a la aparición del caso inicial en la comunidad y si el número reproductivo básico local $\left(\mathrm{R}_{0}\right)$ es menor de 18. Esta estrategia, conocida como profilaxis antiviral dirigida, consiste en el tratamiento de los casos índices iden- 
tificados y de sus contactos, ya sean familiares, vecinos, compañeros de estudio o de trabajo, etc. Además, se recomienda la cuarentena voluntaria en el hogar de los contactos cercanos de los pacientes. Esta estrategia de profilaxis dirigida en el hogar y los hogares vecinos se aplicó en el brote estudiado en Sumatra.

Es muy importante determinar si las posibles cepas pandémicas de gripe son capaces de mantener la transmisión de persona a persona y estimar los parámetros clave de esa transmisión. Es necesario contar con mayor información epidemiológica de la comunidad para poder estimar otros parámetros, además de la tasa de SRA en el hogar. Entre otros datos, se necesita hacer un censo exhaustivo de las personas expuestas en el hogar y en la zona de posible transmisión de la infección, tanto de fuentes animales como humanas. Estos datos permitirían estimar los parámetros de una manera más precisa, entre ellos valores de $\mathrm{R}_{0}$ más exactos.

Los autores desarrollaron un software, TRANSTAT, para realizar estos análisis. Esta aplicación permite la entrada, el almacenamiento y el análisis de los datos de brotes de enfermedades infecciosas agudas. Los métodos estadísticos que utiliza pueden aplicarse en combinación con varias herramientas epidemiológicas convencionales. Esta aplicación permite hacer el análisis en tiempo real y evaluar las medidas de control aplicadas durante un brote, aunque se debe cuidar de introducir tanto los datos de los pacientes como de los contactos. Este sistema está disponible y puede solicitarse directamente a Ira M. Longini, Jr, Program in Biostatistics and Biomathematics, Fred Hutchinson Cancer Research Center, Seattle, WA 98109-1024, USA; correo electrónico: longini@ scharp.org. (Yang Y, Halloran ME, Sugimoto JD, Longini IM. Detecting human-to-human transmission of avian influenza A (H5N1). Emerg Infect Dis. 2007;13(9):1348-53.)

\section{Caracterización molecular de aislamientos de Staphylococcus aureus resistentes a la meticilina en Asunción, Paraguay}

La resistencia de bacterias patógenas a diversos antibióticos es un tema de la mayor importancia para la salud pública debido a la creciente incidencia de infecciones que no ceden al tratamiento habitual con antibióticos. Esta situación se hace aun más crítica cuando se trata de bacterias grampositivas de alta virulencia, como Staphylococcus aureus, una de las bacterias patógenas más frecuentemente involucradas en la etiología de diversos procesos infecciosos, que van desde infecciones superficiales de la piel hasta infecciones generalizadas graves que pueden poner en peligro la vida.
En estudios de genética poblacional de cepas de $S$. aureus resistente a la meticilina (SARM) se han identificado cinco grupos principales de clones que conforman cinco complejos clonales (CC), designados CC5, CC8, CC22, CC30 y CC45. Cada CC está compuesto por aislamientos de SARM con tipos relacionados de secuencias de múltiples locus. Entre los de mayor prevalencia se encuentran los clones arcaico (CC8), ibérico (CC8), brasileño (CC8), pediátrico (CC5) y el clon de New York/Japón (CC5). Estos clones se han caracterizado genéticamente según el polimorfismo de la proteína A del gen spa y los diferentes tipos del casete cromosómico mec del estafilococo (SCCmec), que codifica la resistencia a la meticilina. Las cepas de SARM se han diseminado en los hospitales de todo el mundo. Su prevalencia varía de un país a otro, así como también de un hospital a otro dentro de un mismo país.

En este trabajo se colectaron 96 aislamientos de $S$. aureus de 81 pacientes ingresados en el Hospital de Clínicas, en Asunción, Paraguay, entre abril y octubre de 2005. De esos aislamientos, 42 $(43,8 \%)$ eran resistentes a la meticilina. Todos los aislamientos estaban asociados con infecciones intrahospitalarias. De los aislamientos de SARM (uno por paciente), $34(81,0 \%)$ se caracterizaron por métodos microbiológicos y se determinó la presencia del gen de la toxina y el grupo de alelo de su gen regulador $(a g r)$ mediante la reacción en cadena de la polimerasa. Se obtuvieron los 34 patrones de electroforesis de campo pulsante en gel (ECPG), los que se agruparon en seis tipos de patrones (AS1AS6), según el porcentaje de similitud ( $>80 \%)$. Se caracterizó el perfil de resistencia a antibióticos de cada patrón de ECPG.

Los 27 aislamientos de tres de los patrones de ECPG (AS1-AS3) pertenecían al mismo clon de SARM, CC5, más relacionado por sus características con el clon cordobés/chileno (de origen argentino). Este clon comenzó a desplazar al clon brasileño en los hospitales paraguayos en 1999 y dos años después era el predominante.

Los seis aislamientos pertenecientes a los tipos AS5 y AS6 pertenecían a un mismo clon de SARM, esta vez del grupo CC8, relacionado con el clon brasileño. Por su parte, un aislamiento clasificado como AS4 por su patrón en ECPG, se consideró como SARM esporádico, ya que presentaba resistencia variable a la meticilina.

Se concluyó que los aislamientos de SARM en el Hospital de Clínicas de Asunción, Paraguay, pertenecían principalmente al clon cordobés/chileno (grupo CC5). Este clon está relacionado genéticamente con la cepa EMRSA-3 del Reino Unido, por lo que puede tener un ancestro común con el clon pediátrico y con el clon de New York/Japón. El segundo clon más frecuente fue el brasileño (grupo CC8). 
Esta información es de gran utilidad, no solamente para el control de las infecciones hospitalarias más frecuentes en este hospital paraguayo, sino también para los países vecinos ya que, tal como se ha visto, los patógenos responsables de las infecciones nosocomiales no reconocen fronteras y pueden aparecer en breve tiempo en otros países de la Región. Se debe mantener una especial atención sobre estas cepas de SARM, en particular sobre el clon conocido como cordobés/chileno, por la gran capacidad de diseminación que ha demostrado en Paraguay y otros países, donde ha desplazado a las cepas autóctonas. (Mayor L, Ortellado J, Menacho C, Lird G, Courtier C, Gardon C, et al. Molecular characterization of methicillin-resistant Staphylococcus aureus isolates collected in Asunción, Paraguay. J Clin Microbiol. 2007;45(7):2298-300.)

\section{Eficacia de la vacuna contra la gripe en adultos mayores que viven en la comunidad}

La gripe continúa siendo una de las principales causas de morbilidad y mortalidad, especialmente entre los adultos mayores. Cada año, esta enfermedad y sus complicaciones son responsables de cerca de 186000 ingresos hospitalarios por enfermedades respiratorias y cardiovasculares y de 44000 muertes en este grupo de alto riesgo. Las vacunas contra la gripe son seguras y eficaces, y los adultos mayores se encuentran entre los grupos con mayor prioridad de vacunación.

Se necesitan estimados confiables de los beneficios de la vacunación para establecer políticas informadas de asignación de recursos para la vacunación y para identificar las necesidades de nuevas vacunas y de estrategias de prevención y control de la gripe en este grupo de la población. No obstante, la mayoría de los estudios que evalúan la eficacia de la vacunación contra la gripe en los adultos mayores se basan en una o unas pocas temporadas pico de gripe, por lo que sus resultados tienen un corto alcance. La gran variabilidad del virus de la gripe genera cambios en el comportamiento de la enfermedad y en la eficacia de las vacunas - ya sean favorables o desfavorables desde el punto de vista de la salud pública- y puede provocar que el cuadro derivado de esos estudios sea incompleto o totalmente desorientador a mediano y largo plazos. Además, la falta de consistencia en los resultados disponibles puede reducir las posibilidades de agruparlos apropiadamente en estudios aglutinadores.

En este estudio se examinaron los datos de eficacia de la vacunación contra la gripe entre 1990 y 2000 y se analizaron los posibles factores de sesgos y de confusión que pudieron afectar a los resultados.
Se agruparon los datos individuales de 18 cohortes de adultos mayores asociados con una organización de servicios de salud (HMO) de los Estados Unidos de América desde la temporada de 1990-1991 hasta la de 1999-2000 y de dos HMO desde la temporada de 1996-1997 hasta la de 19992000. Cada cohorte contempló los datos de más de 20000 personas en cada una de las 10 temporadas estudiadas. Se empleó la regresión logística para estimar la eficacia de la vacunación en cuanto a evitar la hospitalización - por neumonía o gripe- y la muerte, después de ajustar por importantes covariables. Se analizaron los posibles sesgos y el efecto de factores residuales de confusión.

La observación abarcó un total de 713872 personas-temporada. La mayoría de las enfermedades observadas tuvieron mayores prevalencias en el grupo vacunado que en el de personas sin vacunar. Se encontró una asociación significativa entre la vacunación contra la gripe y la reducción de $27 \%$ en el riesgo de hospitalización por neumonía o gripe (razón de posibilidades [odds ratio, OR] ajustada: 0,73; intervalo de confianza de 95\% [IC95\%]: 0,68 a 0,77) y de $48 \%$ en el riesgo de muerte (OR ajustada: 0,52; IC95\%: 0,50 a 0,55). Los estimados se mantuvieron estables en todos los grupos de edad y subgrupos de riesgo. A pesar de la modelación del efecto de un hipotético factor de confusión no calculado, el análisis de sensibilidad reveló que no se observó una sobreestimación de los resultados obtenidos y se mantuvo la asociación significativa entre la vacunación y la reducción del riesgo de hospitalización y de muerte.

La cepa viral circulante que predominó en 6 de las 10 temporadas de gripe fue del tipo A/H3N2 (1991-1992, 1993-1994, 1996-1997, 1997-1998, 19981999 y 1999-2000), seguida de la cepa tipo B en una temporada (1990-1991), la combinación del tipo A/H3N2 y B en dos temporadas (1992-1993 y 1994-1995) y la combinación de los tipo A/H3N2 y A/H1N1 en una temporada (1995-1996). La correspondencia entre la cepa de vacunación y los tipos circulantes fue excelente en todas las temporadas, excepto en las de 1992-1993 y 1997-1998, cuando circularon variantes de la cepa A/H3N2.

En este estudio, la vacunación de adultos mayores que vivían en la comunidad contra la gripe durante 10 temporadas pico de esa enfermedad se asoció con una considerable reducción en el riesgo de hospitalización por neumonía o gripe y el riesgo de muerte. Estos resultados muestran una importante perspectiva de los beneficios de la vacunación de ese grupo de riesgo de la población, tomando en cuenta tanto la variabilidad anual observada como el comportamiento global a largo plazo.

El elevado número de personas analizadas permitió llegar a estimados de una elevada preci- 
sión, que demostraron los beneficios en todos los subgrupos de la población estudiada, lo que indica que los beneficios de la vacunación se extienden incluso a un sector mucho más amplio de adultos mayores. El hecho de que las muestras analizadas procedieran de organizaciones de tres diferentes zonas geográficas aumenta la posibilidad de que estos resultados representen a poblaciones de otras partes de los Estados Unidos. Sin embargo, los adultos mayores atendidos en las HMO pueden diferir de los que no se atienden en estas organizaciones, tanto por sus características socioeconómicas y étnicas como por su lugar de residencia, y la generalización de estos resultados se debe hacer con cautela.

La reducción del riesgo de hospitalización por neumonía o gripe y de muerte en los adultos mayores que viven en la comunidad se mantuvo incluso durante las dos temporadas en las que no hubo una buena correspondencia entre la cepa circulante y la cepa empleada en la preparación de la vacuna. Por ello los pacientes, los proveedores de servicios de salud y los encargados de elaborar las políticas sanitarias deben renovar sus esfuerzos para mejorar la cobertura de vacunación contra la gripe en los grupos de mayor riesgo. (Nichol KL, Nordin JD, Nelson DB, Mullooly JP, Hak E. Effectiveness of influenza vaccine in the community-dwelling elderly. N Eng J Med. 2007;357(14):1373-81.)

\section{Factores de riesgo de la infestación de viviendas con triatominos en Colombia}

La enfermedad de Chagas o tripanosomiasis, causada por el parásito Trypanosoma cruzi, es la enfermedad transmitida por insectos de mayor incidencia en América Latina.

Algunos estudios han demostrado la relación existente entre las características de la vivienda y el nivel de infestación con triatominos - tanto dentro como en los alrededores del domicilio- y la prevalencia de la enfermedad. En este trabajo se presentan los resultados de un estudio transversal sobre la relación entre la presencia de anticuerpos contra T. cruzi en niños, el nivel de infestación y la calidad de las viviendas, a partir de una muestra inusualmente grande distribuida en una extensa área de Colombia. Este estudio se llevó a cabo entre 1998 y 2001 con métodos estandarizados y es el primero que se realiza con estas características en el norte de América del Sur.

Se evaluaron 41971 viviendas ubicadas en 3375 poblaciones. Estas poblaciones estaban localizadas en 539 municipios de 15 de los 32 departamentos de Colombia. Para probar la asociación entre dos medidas de infestación (haber visto tria- tominos en la vivienda y el envío de ejemplares al equipo de investigación) y 15 factores de riesgo de las viviendas se empleó la regresión logística multifactorial. El riesgo se determinó en relación con viviendas de referencia de $50 \mathrm{~m}^{2}$, con paredes de adobe sin emplastar, techos de paja y sin cobertizos ni animales domésticos, en las que vivían tres personas o menos.

De las 36873 viviendas de donde respondieron a la pregunta sobre si habían visto triatominos en el interior o en los alrededores, $8576(23,3 \%)$ respondieron afirmativamente. La probabilidad de ver triatominos fue mayor en las viviendas con más de siete habitantes (razón de posibilidades [odds ratio, $\mathrm{OR}]=1,24$; intervalo de confianza de 95\% [IC95\%]: 1,11 a 1,39), espacios de almacenamiento elevados (OR = 1,16; IC95\%: 1,03 a 1,32), cobertizo para granos (OR = 1,25; IC95\%: 1,02 a 1,52), gatos $(\mathrm{OR}=1,27$; IC95\%: 1,14 a 1,42$)$ y cerdos $(\mathrm{OR}=$ 1,16; IC95\%: 1,03 a 1,30). El menor riesgo se observó en las casas con paredes de madera $(\mathrm{OR}=0,46$; IC95\%: 0,34 a 0,61 ), paredes totalmente emplastadas (OR $=0,78$; IC95\%: 0,68 a 0,88), techos de tejas $(\mathrm{OR}=0,51$; IC95\%: 0,33 a 0,78$)$ y pisos de losas $(\mathrm{OR}=0,57$; IC95\%: 0,42 a 0,76).

Las especies enviadas con mayor frecuencia a los investigadores fueron: Rhodnius prolixus, de 554 viviendas (1,32\%); Panstrongylus geniculatus, de $220(0,52 \%)$; Triatoma dimidiata, de $205(0,49 \%)$; T. maculata, de $188(0,45 \%) ; R$. pallescens, de 93 $(0,22 \%)$; y $T$. venosa, de $70(0,17 \%)$. Se recibieron ejemplares de otras 13 especies de un total de 74 $(0,19 \%)$ viviendas.

Los factores de riesgo identificados - en correspondencia total con la información disponible acerca de la ecología de los triatominos-fueron: la presencia de animales domésticos y no domésticos (como roedores, ardillas y zarigüeyas), la existencia de almacenes con productos agrícolas y de construcción, y las características constructivas y las condiciones sanitarias deficientes de la vivienda.

Este estudió demostró que un mayor número de personas en la vivienda y la presencia de gatos y cerdos aumentó significativamente el riesgo de infestación. Las construcciones accesorias, como graneros y cobertizos elevados, ofrecen mayores posibilidades de vida y reproducción a los vectores, sin embargo, los mayores valores de OR correspondieron a las condiciones estructurales deficientes de la vivienda. Las viviendas con paredes, pisos y techos construidos con materiales de construcción más sólidos y compactos presentaron un menor riesgo de infestación.

El análisis de los resultados de este estudio hace notar la robustez estadística de la asociación encontrada entre las características de la vivienda y la probabilidad de infestación doméstica con triato- 
minos. Además, demuestra que la participación comunitaria puedes ser de gran ayuda para mapear y caracterizar la diseminación de los triatominos en extensas zonas y para identificar los factores de riesgo en las viviendas y sus alrededores. Estos resultados son de particular interés no solo para Colombia, sino también para otros países vecinos donde habitan los mismos vectores y las viviendas tienen características similares. (Campbell-Lendrum DH, Angulo VM, Esteban L, Tarazona Z, Parra GJ, Restrepo $\mathrm{M}$, et al. House-level risk factors for triatomine infestation in Colombia. Intl J Epidemiol. 2007; 36(4):866-72.)

\section{La gripe en Estados Unidos de 2006 a 2007 y la vacuna para 2007 a 2008}

En el período del 1 octubre de 2006 al 19 de mayo de 2007, la OMS y sus laboratorios colaboradores en los Estados Unidos (Sistema Nacional de Vigilancia de Virus Respiratorios y Entéricos) examinaron 179268 especímenes de virus y 23753 $(13,2 \%)$ dieron resultados positivos de gripe. De estos, $18817(79,2 \%)$ correspondieron al serotipo A y $4936(20,8 \%)$, al B. La proporción de positivos sobrepasó el 10\% la semana que terminó el 23 de diciembre de 2006, llegó al máximo de $28,6 \%$ en la semana que terminó el 10 de febrero de 2007 y bajó a menos de $10 \%$ en la semana que terminó el 28 de abril de 2007. Durante las tres temporadas anteriores, el porcentaje de especímenes positivos osciló de $22,6 \%$ a 34,7\% y el valor máximo se manifestó desde principios de diciembre hasta marzo. Durante 13 a 17 semanas consecutivas hubo más de $10 \%$ de resultados positivos, pero el porcentaje de defunciones por gripe y neumonía permaneció por debajo de los valores iniciales. Los virus A (H1) predominaron en esa temporada, si bien los A (H3) se aislaron con mayor frecuencia desde principios de marzo y predominaron en muchos países europeos y asiáticos.

En los Estados Unidos, los epidemiólogos informan de la distribución de la gripe en su propio estado o territorio mediante un código de actividad semanal de la enfermedad. Su mayor difusión se notó en la octava semana de 2007, cuando se extendió a 25 estados y 19 notificaron actividad regional. En 41 estados hubo actividad gripal dispersa por lo menos en una ocasión durante la temporada, pero en las semanas 16 a 20 de 2007 (en abril y mayo) la actividad de la gripe disminuyó. Durante las tres temporadas anteriores, el mayor número de estados informantes había variado de 41 a 50 . La mayor parte de los virus A (H1) se caracterizaron como A/Nueva Caledonia/20/99, que fue el componente de A (H1N1) recomendado para la vacuna de 200607. La mitad de los virus B del linaje B/Victoria se caracterizaron como B/Ohio/01/2005, el cual antigénicamente equivale al B/Malasia/2506/2004, componente $\mathrm{B}$ recomendado para la misma vacuna.

En los primeros meses de la temporada 200607, la mayor parte de los aislados A (H3) eran próximos a la cepa A/Wisconsin/67/2005, la A (H3N2) recomendada para inclusión en la vacuna. A fines de febrero de 2007, la mayor parte de aislados A (H3) mostraban títulos reducidos con antisueros producidos contra la cepa A/Wisconsin/67/2005.

En mayo de 2007, los Centros para el Control y la Prevención de Enfermedades (CDC) difundieron el aviso de la Red de Alerta de Salud de que había aumentado el número de defunciones pediátricas asociadas con gripe y coinfección por Staphylococcus aureus. En 2004-05 hubo solo una defunción pediátrica por esa causa, pero en 2005-06 se supo de tres defunciones. Durante el período del 1 de octubre de 2006 al 19 de mayo de 2007 ocurrieron 68 muertes infantiles asociadas con infecciones gripales, de las cuales 21 eran coinfecciones de gripe con S. aureus, tanto resistente como sensible a la meticilina.

En junio de 2007, en la reunión anual del Consejo de Epidemiólogos Estatales y Territoriales (CSTE), se ratificó la obligatoriedad de notificar al Sistema Nacional de Vigilancia de las Enfermedades las infecciones humanas por virus A novedosos. Estos se definieron como virus aislados de personas pero subtipificados como no humanos o virus que no pueden subtipificarse con los métodos estándar. Virus novedosos transmisibles de persona a persona podrían significar el principio de una pandemia y su pronta detección y caracterización aceleraría la ejecución de medidas protectoras de salud pública. También se mantuvo obligatoria la notificación al Sistema de Vigilancia de muertes pediátricas relacionadas con la gripe. Asimismo, los CDC siguen recomendando una vigilancia intensificada para detectar cualquier caso posible de gripe aviar A (H5N1) en viajeros con trastornos respiratorios importantes de causa desconocida, que hayan regresado al país hace poco tiempo tras visitar países afectados.

Sobre la base de análisis antigénicos de los virus de gripe aislados recientemente, datos epidemiológicos, estudios serológicos posvacunales y la disponibilidad de cepas de vacuna y reactivos, la vacuna trivalente contra la gripe recomendada para 2007-08 contiene cepas análogas a la A/Islas Salomón/3/2006 (H1N1), la A/Wisconsin/67/ 2005 (H3N2) y la B/Malasia/2506/2004. El único cambio en esta vacuna es el componente del virus A (H1N1). La cepa A/Islas Salomón/3/2006 es una variante antigénica reciente de la $\mathrm{A} / \mathrm{Nueva}$ Caledonia/20/99 usada en la vacuna 2006-07. (Centers for Disease Control and Prevention. Morb Mortal Wkly Rep. 2007;56(31):789-94.) 